2. - Sur les spectres des fonctions, Congrès sur L'Analyse Harmonique, Nancy, 1947.

3. Y. Domar, Harmonic analysis based on certain commutative Banach algebras, Acta Math. 96 (1956), 2-66.

4. N. Dunford, Spectral theory. II. Resolutions of the identity, Pacific J. Math. 2 (1952), 559-614.

5. - Spectral operators, Pacific J. Math. 4 (1954), 321-354.

6. G. Leaf, $A$ spectral theory for a class of linear operators, Pacific J. Math. 13 (1963), 141-155.

7. E. R. Lorch, The integral representation of weakly almost periodic transformations in reflexive vector spaces, Trans. Amer. Math. Soc. 49 (1941), 18-40. 622.

8. J. Wermer, The existence of invariant subspaces, Duke Math. J. 19 (1952), 615-

9. F. Wolf, Operators in Banach space which admit a generalized spectral decomposition, Proc. Akad. Wetensch. Ser. A 60 (1957), 302-311.

Argonne National Laboratory, Argonne, Illinois

\title{
A NOTE ON NORMAL DILATIONS
}

\section{ARNOLD LEBOW}

I. Introduction. Our purpose is to give certain sufficient conditions that a normal dilation of an operator be an extension from a reducing subspace. The first result of this kind, that we know of, is due to T. Andô [1] who considered compact normal dilations. In this note we use only assumptions about the nature of the spectrum; nevertheless, we are able to recover Andô's theorem.

Let $A$ be an operator on a Hilbert space $\Re$. Let $P$ be the orthogonal projection of $\nVdash$ onto a subspace $\Re$. Let $T$ denote the restriction of $P A P$ to $\mathcal{F C}$. The operator $T$ is called a compression of $A$ and $A$ is called a dilation of $T$. If $T^{n}$ is the compression of $A^{n}(n=01,2,3, \cdots)$ then $T$ is called a strong compression and $A$ a strong dilation. Let $X$ be a compact subset of the plane containing $\sigma(A)$ and $\sigma(T)$, the spectra of $A$ and $T$. The operator $A$ is said to be an $X$-dilation of $T$ if, for every rational function $r(\cdot)$ which is analytic on $X$, the operator $r(A)$ is a dilation of $r(T)$. These definitions were introduced by Halmos. Some other writers use "dilation" to mean what we call strong dilation. Sz-Nagy uses "projection" for compression. When $T$ is a strong compression of $A$ Andô calls $\mathfrak{H}$ a "semi-invariant" subspace of $A$.

These notions are related to the more familiar concepts of invariant subspace and reducing subspace as follows. If $\mathcal{T C}$ is an invariant sub-

Presented to the Society, January 27, 1965 under the title On a theorem of Andô; received by the editors September 21, 1964. 
space (equivalently $P A P=A P$ ) and $X$ contains $\sigma(A)$ and $\sigma(T)$ then $A$ is an $X$-dilation of $T$. If $\xi \mathcal{C}$ is reducing and $X$ contains $\sigma(A)$ then $A$ is an $X$-dilation of $T$. When $\mathcal{H}$ is invariant, we say $A$ is an extension of $T$; when $\mathfrak{H C}$ is reducing, we say $A$ is a direct extension of $T$ and $T$ is a reduction of $A$.

Summing up, we have five conditions:

(i) $A$ is a dilation of $T$;

(ii) $A$ is a strong dilation of $T, P A^{n} P=(P A P)^{n}$;

(iii) $A$ is an $X$-dilation of $T, \operatorname{Pr}(A) P=r(P A P)$;

(iv) $A$ is an extension of $T, P A P=A P$; and

(v) $A$ is a direct extension of $T, A P=P A$.

These are also expressed as: $T$ is a (i) compression, (ii) strong compression, (iii) $X$-compression, (iv) restriction, and (v) reduction of $A$. One sees at once

$$
\text { (v) } \Rightarrow \text { (iv) } \Rightarrow \text { (iii) } \Rightarrow \text { (ii) } \Rightarrow \text { (i) }
$$

There are examples which show that no arrow can be reversed unless more assumptions are made.

It is known [2, p. 60] that if both $T$ and $A$ are normal, then (iv) implies (v). T. Andô [1] has proved that if $A$ is compact and normal, then (v) follows from (ii). It will be shown here that we need only assume that $A$ is normal and $\sigma(A)$ is nowhere dense and does not separate the plane. This result depends on the density of the set of polynomials in the space, $C(\sigma(A))$, of complex continuous functions on $\sigma(A)$. If the set of rational functions is dense in $C(X)$, then (iii) implies (v).

\section{Statement and proof of results.}

REMARK (Andô). Let $E$ and $P$ be projections. The operator $Q=P E P$ is a projection if and only if $P$ and $E$ commute.

Proof. If $Q$ is a projection we have that $\|E P x-Q x\|^{2}=0$, by expansion to a sum of inner products. Now note

$$
E P=Q=Q^{*}=(E P)^{*}=P^{*} E^{*}=P E .
$$

The converse is trivial.

Lemma. Let $T$ be a normal operator with a normal dilation $A$, and $X$ be a compact set containing $\sigma(A)$ and $\sigma(T)$.

If the set

$$
D=\{f \in C(X) \mid f(A) \text { is a dilation of } f(T)\}
$$

is dense in $C(X)$ then $T$ is a reduction of $A$. 
Proof. For every pair of vectors $x$ in $\mathcal{K}$ and $y$ in $\mathcal{K}$, if $f \in D$ we have (1) $(f(A) x, P y)=\left(f(A) P x, P^{2} y\right)=(P f(A) P x, P y)=(f(T) x, P y)$.

Denoting the spectral measures of $A$ and $T$ by $E(\cdot)$ and $F(\cdot)$, respectively, (1) may be written as

$$
\int f(\lambda) d(E(\lambda) x, P y)=\int f(\lambda) d(F(\lambda) x, P y) .
$$

Since $D$ is dense in $C(X)$ and the measures $(E(\cdot) x, P y)$ and $(F(\cdot) x$, $P y)$ are regular, it follows that they are identical. That is for each Borel set $\delta$

$$
(E(\delta) x, P y)=(F(\delta) x, P y)
$$

and therefore

$$
(P E(\delta) x, y)=(F(\delta) x, y) .
$$

Since $y$ is arbitrary $P E(\delta) x=F(\delta) x$ for all $x$ in $\mathcal{F}$. This means that $P E(\delta) P$ acts as a projection in $\mathfrak{H C}$ and since $P E(\delta) P$ acts as 0 in $\mathcal{F}^{\perp}$ it is evident that $P E(\delta) P$ is a projection. It follows from the Remark that $P$ commutes with $E(\delta)$ and from the spectral theorem that $P$ commutes with $\int \lambda d E(\lambda)=A$.

Theorem 1. Let $A$ be a normal strong dilation of $T$. If $\sigma(A)$ is nowhere dense and does not separate the plane, then $T$ is a reduction of $A$.

Proof. Since $T$ is a strong dilation

$$
(P A P)^{n}=P A^{n} P ; \quad n=1,2,3, \cdots,
$$

for every polynomial $p(\cdot), P A P$ commutes with $P p(A) P$. By Lavrentieff's theorem [4] the polynomials are dense in $C(\sigma(A))$ and thus the functional calculus for normal operators implies that $A^{*}$ is in the uniform closure of $\{p(A)\}$, and so $P A^{*} P$ is in the uniform closure of $\{P p(A) P\}$. Hence $P A P$ commutes with $P A^{*} P$ and is therefore normal. The operator $T$ is normal because it is the reduction of the normal operator $P A P$.

Now with $D$ being the set of polynomials and $X=\sigma(A)$ it follows from the Lemma that $T$ is a reduction of $A$.

CoROLlARY (ANDô). Every strong compression of a compact normal operator is a reduction.

The method for proving Theorem 1 can be used to obtain other similar results. We shall refrain from maximum generality and will be satisfied to state one theorem on $X$-dilations. If the rational functions with poles not in $X$ are dense in $C(X)$ we call $X$ an R-set. Bishop [3] 
has given a characterization of $\mathbb{R}$-sets which includes the HartogsRosenthal result that every set of zero planar Lebesgue measure is an R-set. Mergelyan [4] has obtained other sufficient conditions $X$ be an $R$-set.

Theorem 2. If $X$ is an R-set and $T$ has a normal $X$-dilation $A$, then $T$ is a reduction of $A$.

The proof follows the outlines of that of Theorem 1 .

As one more variation on this theme we state:

Theorem 3. If $A^{n}$ is a normal dilation of $T^{n}$ for $n=1,2,3, \cdots, k$ and $\sigma(A)$ is a finite set of not more than $k$ points, then $T$ is a reduction of $A$.

Note that if $k=2$ and $A$ is a projection, Theorem 3 reduces to the Remark at the beginning of this section.

Donald Sarason has informed the author (personal communication) that he has obtained Theorems 1 and 2 by different methods.

\section{BIBLIOGRAPHY}

1. T. Ando, $A$ note on invariant subspaces of normal operators, Arch. Math. 14 (1963), 337-340.

2. S. K. Berberian, Introduction to Hilbert space, Oxford Univ. Press, New York, 1961.

3. E. Bishop, $A$ minimal boundary for function algebras, Pacific J. Math. 11 (1959), 629-642.

4. S. N. Mergelyan, Uniform approximations to functions of a complex variable, Amer. Math. Soc. Transl. (1) 3 (1954), 294-391.

New York UNIVERSITY 